\title{
Inferior outcome of allogeneic stem cell transplantation for secondary acute myeloid leukemia in first complete remission as compared to de novo acute myeloid leukemia
}

\author{
Ann-Kristin Schmaelter ${ }^{1}$, Myriam Labopin ${ }^{2,3}$, Gerard Socié $\mathbb{0}^{4}$, Maija Itälä-Remes ${ }^{5}$, Didier Blaise (0) \\ Ibrahim Yakoub-Agha ${ }^{7}$, Edouard Forcade ${ }^{8}$, Jan Cornelissen ${ }^{9}$, Arnold Ganser $^{10}$, Dietrich Beelen ${ }^{11}$, \\ Hélène Labussière-Wallet ${ }^{12}$, Jakob Passweg ${ }^{13}$, Bipin N. Savani (1) ${ }^{14}$, Christoph Schmid (1) ${ }^{1}$, Arnon Nagler ${ }^{3,15}$ and \\ Mohamad Mohty ${ }^{2}$
}

\begin{abstract}
Following chemotherapy, secondary acute myeloid leukemia (sAML), occurring after antecedent hematologic diseases, previous chemotherapy or radiation, has an inferior prognosis compared with de novo AML. To define the outcome of SAML in the context of allogeneic stem cell transplantation (alloSCT), a retrospective, registry-based comparison was performed, including 11,439 patients with de novo and 1325 with sAML. Among transplants in first complete remission (CR1) $(n=8,600)$, the 3-year cumulative incidence of relapse (RI) and non-relapse mortality (NRM) was $28.5 \%$ and $16.4 \%$ for de novo, and $35 \%$ and $23.4 \%$ for sAML. Three-year overall survival (OS), leukemia-free survival (LFS) and Graft-versus-Host Disease/relapse-free survival (GRFS) was 60.8\%, 55.1\%, and 38.6\% for de novo, and 46.7\%, 41.6\%, and $28.4 \%$ for $\mathrm{SAML}$, respectively. In multivariate analysis, sAML was associated with a lower OS $(\mathrm{HR}=1.33[95 \% \mathrm{Cl}=$ $\left.1.21-1.48] ; p<10^{-5}\right)$, LFS $\left(H R=1.32[95 \% \mathrm{Cl}=1.19-1.45] ; p<10^{-5}\right)$ and $\operatorname{GRFS}\left(\mathrm{HR}=1.2[95 \% \mathrm{Cl}=1.1-1.31] ; p<10^{-4}\right)$ and higher NRM $\left(H R=1.37[95 \% \mathrm{Cl}=1.17-1.59] ; p<10^{-4}\right)$ and $\mathrm{RI}\left(\mathrm{HR}=1.27[95 \% \mathrm{Cl}=1.12-1.44] ; p<10^{-3}\right)$. Results of the Cox model were confirmed in a matched-pair analysis. In contrast, results did not differ between de novo and SAML after alloSCT in induction failure or relapse. Hence, this analysis identified SAML as an independent risk factor for outcome after alloSCT in CR1.
\end{abstract}

\section{Introduction}

Secondary acute myeloid leukemia (sAML) arises from underlying hematological disorders such as myelodysplastic syndrome (MDS), myeloproliferative neoplasm (MPN), MPN/MDS overlap or bone marrow failure syndromes. Furthermore, therapy related AML (tAML),

Correspondence: Christoph Schmid (Christoph.Schmid@uk-augsburg.de) 'Department of Hematology and Oncology, Augsburg University Hospital, Augsburg, Germany

${ }^{2}$ Department of Haematology, Saint Antoine Hospital, Université Pierre et Marie Curie, INSERM UMR 938 Paris, France

Full list of author information is available at the end of the article. which can develop after a prior treatment by chemotherapy or radiation, can be assigned to the sAML classification ${ }^{1}$.

Treating SAML with conventional chemotherapy or autologous stem cell transplantation is associated with a poor prognosis ${ }^{2-4}$. Therefore, in comparison to de novo AML, sAML is thought to have an inferior prognosis in general $^{5-7}$. Allogeneic stem cell transplantation (alloSCT) is regarded as the treatment option for sAML with the best chance of achieving a long-term remission. Considering the transplant setting ${ }^{8,9}$, patients with sAML tend to have less available HLA identical siblings, and the

\section{(c) The Author(s) 2020}

(c) (i) Open Access This article is licensed under a Creative Commons Attribution 4.0 International License, which permits use, sharing, adaptation, distribution and reproduction in any medium or format, as long as you give appropriate credit to the original author(s) and the source, provide a link to the Creative Commons license, and indicate if changes were made. The images or other third party material in this article are included in the article's Creative Commons license, unless indicated otherwise in a credit line to the material. If material is not included in the article's Creative Commons license and your intended use is not permitted by statutory regulation or exceeds the permitted use, you will need to obtain permission directly from the copyright holder. To view a copy of this license, visit http://creativecommons.org/licenses/by/4.0/. 
majority receive reduced intensity conditioning (RIC) ${ }^{10,11}$. However, it is not clear whether having sAML per se is a risk factor for outcome after alloSCT, when all wellknown risk factors are controlled for ${ }^{8}$.

So far, only smaller studies have addressed this question $^{12}$. Therefore, the Acute Leukemia Working Party (ALWP) of the European Society of Blood and Marrow Transplantation (EBMT) performed a retrospective registry-based analysis, comparing the outcome of adult patients diagnosed with sAML and de novo AML, who had received alloSCT.

\section{Methods}

\section{Study design}

Data were provided by the EBMT registry, which comprises more than 600 transplant centers providing reports and annual follow-up on all consecutive stem cell transplantations. Audits are routinely performed to determine the accuracy of the data. Since 1990, patients have provided informed consent, authorizing the use of their personal information for research purposes. The study was approved by the general assembly and review board of the ALWP.

Inclusion criteria were age $\geq 18$ years, alloSCT between January 2000 and December 2016 from a matched related, $9 / 10$ or $10 / 10$ antigen matched unrelated, or a T-cell replete haploidentical donor for either de novo or sAML, and available information on cytogenetics. The number of patients fitting inclusion criteria was 11,439 patients with de novo AML and 1325 with sAML.

Variables of interest were patient and donor characteristics (age, gender, Karnofsky performance score [KPS], cytomegalovirus [CMV] serostatus), diseaserelated (favorable/intermediate/adverse cytogenetics according to the British Medical Research Council classification ${ }^{13}$, remission status at alloSCT) and transplantrelated factors (graft source, donor type, conditioning, Tcell depletion [TCD] and graft-versus-host disease [GVHD] prophylaxis). Outcomes comprised overall survival (OS), leukemia free survival (LFS), GVHD/relapsefree survival (GRFS), cumulative incidence of relapse (RI), non-relapse mortality (NRM), acute and chronic GVHD, and cause of death.

\section{Definitions}

Secondary AML was defined as AML with an antecedent MDS, MPN or other malignant hematologic disorder, bone marrow failure syndrome, or solid tumor with prior chemotherapy or radiation ${ }^{8}$. For a subgroup analysis, tAML was defined by previous treatment of solid tumors, Hodgkin or non-Hodgkin Lymphoma (NHL), acute lymphoblastic leukemia (ALL) or chronic lymphocytic leukemia (CLL) using chemotherapy or radiation ${ }^{14}$.
As recommended complete remission (CR) was defined by $<5 \%$ bone marrow (BM) blasts, absence of circulating blasts and extramedullary disease. Failure to achieve CR after two courses of standard induction chemotherapy was defined as primary induction failure (PIF). Relapse was defined by more than $5 \%$ BM blasts or reappearance of circulating blasts after a documented $\mathrm{CR}^{15}$. OS was defined as the interval between day of alloSCT and day of death or last follow-up, LFS as interval between alloSCT and date of leukemia persistence, relapse or progression. NRM was defined as death from any cause without relapse or progression. GRFS was defined as absence of acute GVHD III-IV, chronic GVHD requiring systemic treatment, relapse, or death ${ }^{16,17}$. Reduced intensity conditioning (RIC) was defined using EBMT criteria $^{18}$. Cytogenetic subgroups were defined according to SWOG criteria $^{19}$.

\section{Statistical analysis}

Descriptive statistics used median, inter-quartile range (IQR), minimum (min) and maximum (max) for continuous data, counts and percentages for categorical variables. Patient, disease, and transplant-related characteristics for the two cohorts (de novo or secondary AML) were compared by using $X^{2}$ statistics for categorical variables and the Mann-Whitney test for continuous variables. The date of transplantation was the starting point for time-to-event analysis. Survivors were censored at last contact. Cumulative incidence was used to estimate the endpoints of NRM, RI, acute and chronic GVHD to accommodate for competing risks. Relapse and death were considered competing events for acute and chronic GVHD. Probabilities of OS, LFS, and GRFS were calculated using the Kaplan-Meier method. Univariate analyses were done using the Gray's test for cumulative incidence functions and the log rank test for OS, GRFS, and LFS.

A multivariate Cox proportional-hazards model was performed to account for imbalances of risk factors between the two cohorts. Due to a significant interaction between disease stage at transplant and diagnosis (de novo or SAML), the analysis was stratified by stage at alloSCT. All variables associated with a significant outcome in univariate analysis $(p<0.05)$, factors known from the literature to have a potential influence on outcome, and variables not equally balanced between cohorts were included in the Cox model.

To confirm the results from the Cox model, a matchedpair analysis of secondary versus de novo AML was performed, using the following criteria for matching: Age $( \pm 3$ years), cytogenetics, stage at alloSCT, KPS, conditioning, in and ex vivo TCD, donor type, donor/recipient gender and CMV serostatus combinations, and graft source. 
Results were expressed as hazard ratio (HR) and 95\% confidence intervals (CI). Proportional hazards assumptions were checked for all models using the GrambschTherneau residual-based test. All tests were 2 -sided. The Type I error rate was fixed at 0.05 for the determination of factors associated with time-to-event outcomes. Statistical analyses were performed with SPSS 24.0 (SPSS Inc, Chicago, IL) and R 3.4.1 (https://www.R-project.org/).

\section{Results}

\section{Patients' disease and transplant characteristics}

Using the criteria defined above, 11,439 patients with de novo AML and 1325 with sAML were included. Median follow-up after alloSCT was 36.1 and 33.1 months for de novo and sAML, respectively. Patients' disease- and transplant-related characteristics are outlined in Table 1. Among patients with sAML, 825 (62.5\%) had evolved from previous myeloid malignancy (most frequently MDS [68.5\%], MPN [11.0\%], and MDS/MPN overlap [13.3\%]) or bone marrow failure syndrome. In 500 (37.7\%) patients sAML was treatment related, following chemotherapy or radiation for other types of cancer, with breast cancer (38.2\%) being the most frequent malignancy, followed by lymphoma (33.2\%), other solid tumors (26.2\%), ALL (2.4\%), and CLL and myeloma ( $<1 \%$ each). In the de novo AML and sAML cohorts 6306 (55.1\%) and 574 (43.3\%), respectively, received a myeloablative conditioning regimen (MAC), whereas 5,133 (44.9\%) of de novo AML patients and $751(56.7 \%)$ of sAML patients were treated with a RIC regimen (cf. supplemental Tables 1-2 for details on conditioning and immunosuppression).

\section{Outcomes}

Engraftment was achieved in 11,133 (98.2\%) and 1264 (96.6\%) patients with de novo AML and sAML, respectively. Three-year OS and LFS rates for the entire cohort were 54.6\% [53.6-55.6] and 48.9\% [47.9-49.9] for de novo AML and 43.1\% [40.1-46] and 37.9\% [35.1-40.8] for sAML. Three-year NRM, RI, and GRFS were $18 \%$ [17.3-18.8], 33\% [32.1-34], and 34.3\% [33.3-35.3] respectively, for de novo and $24.8 \%$ [22.4-27.3], 37.3\% [34.5-40.1], and $25.8 \%[23.1-28.4]$, respectively, for sAML. Overall incidence of acute GvHD grades II-IV and chronic GvHD were 23.5\% [22.7-24.3]/40.6\% [39.6-41.6] for de novo AML and 24.4\% [22-26.8]/35.4\% [32.6-38.1] for sAML. The most frequent cause of death was the reoccurrence of the original disease, other causes of death are shown in supplemental Table S3.

\section{Comparison between de novo and secondary AML}

As mentioned above, a significant interaction between disease status at transplant and diagnosis (de novo or sAML) was observed. Therefore, the analysis of the role of sAML had to be stratified according to disease status at
Table 1 Patient, disease and transplant characteristics in entire population.

\begin{tabular}{llll}
\hline Characteristic & de novo AML & sAML & $p$ \\
\hline N & $11439(89.62 \%)$ & $1325(10.38 \%)$ & \\
Age at SCT (year), & $49.3(18-76.8)$ & $57.7(18.3-76)$ & $<10^{-3}$ \\
median, (range) (IQR) & $(37.8-58.2)$ & $(49.3-63.5)$ & \\
Year of SCT, median & 2011 & 2012 & $<10^{-3}$ \\
(range) (IQR) & $(2000-2016)$ & $(2000-2016)$ & \\
& $(2007-2014)$ & $(2009-2014)$ & \\
Months diagnosis to & $5.6(4.1-9.7)$ & $4.7(3.5-6.3)$ & $<10^{-3}$ \\
SCT, median (IQR) & & &
\end{tabular}

Status at SCT

\begin{tabular}{|c|c|c|c|}
\hline CR1 & $7691(67.23 \%)$ & $909(68.6 \%)$ & $<10^{-3}$ \\
\hline CR2 & $2132(18.64 \%)$ & $93(7.02 \%)$ & \\
\hline PIF & $607(5.31 \%)$ & 199 (15.02\%) & \\
\hline Relapse & $1009(8.82 \%)$ & $124(9.36 \%)$ & \\
\hline \multicolumn{4}{|l|}{ Donor to patient sex } \\
\hline No female to male & $9188(80.32 \%)$ & $1096(82.72 \%)$ & \multirow[t]{2}{*}{0.037} \\
\hline Female to male & $2251(19.68 \%)$ & $229(17.28 \%)$ & \\
\hline \multicolumn{4}{|l|}{ KPS at SCT } \\
\hline $\mathrm{KPS}<80$ & $553(4.83 \%)$ & $94(7.09 \%)$ & \multirow[t]{2}{*}{$<10^{-3}$} \\
\hline KPS $\geq 80$ & $10,886(95.17 \%)$ & $1231(92.91 \%)$ & \\
\hline \multicolumn{4}{|l|}{ Donor } \\
\hline MSD & 6405 (55.99\%) & $578(43.62 \%)$ & \multirow[t]{4}{*}{$<10^{-3}$} \\
\hline UD 10/10 & $3281(28.68 \%)$ & $518(39.09 \%)$ & \\
\hline UD 9/10 & 1035 (9.05\%) & 139 (10.49\%) & \\
\hline Haploidentical donor & $718(6.28 \%)$ & $90(6.79 \%)$ & \\
\hline \multicolumn{4}{|c|}{ CMV status donor (D) and recipient (R) } \\
\hline $\mathrm{D}-/ \mathrm{R}-$ & $2725(23.82 \%)$ & $300(22.64 \%)$ & \multirow[t]{4}{*}{0.005} \\
\hline$D+/ R-$ & $1060(9.27 \%)$ & $112(8.45 \%)$ & \\
\hline $\mathrm{D}-/ \mathrm{R}+$ & $2524(22.06 \%)$ & $350(26.42 \%)$ & \\
\hline $\mathrm{D}+/ \mathrm{R}+$ & $5130(44.85 \%)$ & $563(42.49 \%)$ & \\
\hline \multicolumn{4}{|l|}{ Cytogenetics } \\
\hline Favorable & 1492 (13.04\%) & $58(4.38 \%)$ & \multirow[t]{3}{*}{$<10^{-3}$} \\
\hline Intermediate & 7809 (68.27\%) & 836 (63.09\%) & \\
\hline Adverse & 2138 (18.69\%) & 431 (32.53\%) & \\
\hline \multicolumn{4}{|l|}{ Graft source } \\
\hline Bone marrow & 2005 (17.53\%) & $162(12.23 \%)$ & \multirow[t]{2}{*}{$<10^{-3}$} \\
\hline Peripheral blood & 9431 (82.47\%) & 1163 (87.77\%) & \\
\hline \multicolumn{4}{|l|}{ Conditioning regimen } \\
\hline MAC & 6306 (55.13\%) & $574(43.32 \%)$ & \multirow[t]{2}{*}{$<10^{-3}$} \\
\hline $\mathrm{RIC}$ & 5133 (44.87\%) & 751 (56.68\%) & \\
\hline
\end{tabular}


Table 1 continued

\begin{tabular}{llll}
\hline Characteristic & de novo AML & sAML & $p$ \\
\hline T-cell depletion (TCD) & & & \\
No in vivo TCD & $5951(52.02 \%)$ & $502(37.89 \%)$ & $<10^{-3}$ \\
In vivo TCD & $5488(47.98 \%)$ & $823(62.11 \%)$ & \\
No ex vivo TCD & $10964(95.85 \%)$ & $1305(98.49 \%)$ & $<10^{-3}$ \\
Ex vivo TCD & $475(4.15 \%)$ & $20(1.51 \%)$ & \\
\hline
\end{tabular}

Graft source: 3 missing in de novo AML group.

Details on conditioning regimes are presented in supplemental Table S1.

Details on immunosuppression are presented in supplemental Table S2.

SCT stem cell transplantation, IQR interquartile range, CR complete remission, PIF primary induction failure, KPS Karnofsky performance status, MSD matched sibling donor, UD unrelated donor, CMV cytomegalovirus, MAC myeloablative conditioning, RIC reduced-intensity conditioning, TCD T-cell depletion.

time of alloSCT. We focused on the comparison of patients transplanted in CR1, primary refractory phase and relapse, as only 93 patients with sAML were reported to have been transplanted in CR2, precluding relevant adjustment in that subgroup.

\section{Transplantation in first complete remission}

Transplantation in CR1 had been performed in 7691 (67.2\%) patients with de novo and 909 (68.6\%) with sAML. Three-year cumulative RI and NRM after alloSCT was $28.5 \%$ and $16.4 \%$ for de novo AML and $35 \%$ and $23.4 \%$ for sAML, respectively. Three-year OS, LFS and GRFS were $60.8 \%, 55.1 \%$, and $38.6 \%$, respectively, for de novo and $46.7 \%, 41.6 \%$, and $28.4 \%$, respectively, for sAML (all $p$-values $<10^{-5}$ ) (Fig. 1, Table 2).

In a multivariate Cox model, sAML was associated with a lower OS $\left(\mathrm{HR}=1.33[1.21-1.48] ; p<10^{-5}\right)$. Other prognostic factors for OS after alloSCT in CR1 were age $\left(\mathrm{HR}=1.19[1.15-1.23] ; p=<10^{-5}\right)$, cytogenetics (intermediate, $\mathrm{HR}=1.27$ [1.09-1.48]; $p=0.002$, adverse, $\mathrm{HR}=$ 2 [1.7-2.35]; $p=\left\langle 10^{-5}\right.$ ), patient/donor sex combination (female in male, HR $=1.12$ [1.03-1.22]; $p=0.007$ ), KPS > $80 \%\left(\mathrm{HR}=0.66[0.56-0.79] ; p<10^{-5}\right)$ and donor type $(10 / 10$ matched unrelated donor [UD], $\quad H R=1.14$ [1.04-1.24]; $p=0.007 ; 9 / 10$ matched UD, $\mathrm{HR}=1.36$ [1.19-1.56]; $p<10^{-5}$; haploidentical donor, $\mathrm{HR}=1.33$ [1.11-1.6]; $p=0.002$ ) (Table 3).

Regarding other outcome parameters, multivariate analysis showed that SAML was associated with a lower LFS (HR $\left.=1.32[1.19-1.45] ; p<10^{-5}\right)$ and GRFS (HR $=$ $\left.1.2[1.1-1.31] ; \quad p<10^{-4}\right)$, a higher NRM $(\mathrm{HR}=1.37$ $\left.[1.17-1.59] ; p<10^{-3}\right)$ and RI (HR $=1.27$ [1.12-1.44]; $p<$ $\left.10^{-4}\right)$. Detailed results of a Cox model of risk factors for RI, NRM, LFS, and GRFS are shown in supplemental Tables 4-7. The inferior outcome of patients with sAML was also observed in an exploratory subgroup analysis of patients below and above the age of 60 years (data not shown).

\section{Separate comparison for AML evolving from myeloid malignancies/bone marrow failure syndromes and treatment-related AML}

To evaluate a potential difference among patients with sAML evolving from myeloid malignancies or bone marrow failure syndromes versus treatment-related AML, a second Cox model was fitted, including the two subtypes of sAML as covariates. Both sAML after myeloid malignancies/BM failure and tAML were associated with increased OS, LFS, and GRFS following alloSCT in CR1 when compared to de novo AML (supplemental Table S8).

\section{Transplantation in primary induction failure or active relapse}

Among patients with sAML, 199 (15.02\%) and 124 (9.36\%) had been transplanted in PIF and relapsed disease, respectively; in de novo AML, 607 (5.3\%) had PIF, and $1009(8.82 \%)$ had relapsed disease at time of alloSCT. In contrast to the cohort transplanted in CR1, sAML was not identified as a relevant factor for OS (PIF: $\mathrm{HR}=0.92$; $p=$ 0.465 ; relapse: $\mathrm{HR}=0.95 ; p=0.64$; cf. Tables 2 and 3 ), nor for RI, NRM, LFS, and GRFS. Risk factors that affected OS, RI, LFS, and GRFS in the cohort transplanted for active disease included cytogenetics and KPS $>80 \%$ (cf. Table 3 and supplemental Tables 4-7 for details).

\section{Role of SAML for GVHD}

Independently of disease status before transplantation, sAML was not a risk factor for the occurrence of acute or chronic GVHD. Risk factors for acute GVHD grade II-IV across all subgroups were donor type, female donor for male patient and in vivo TCD. In the CR1 cohort, among others, age, cytogenetics, KPS, and conditioning regime were additional factors. Risk of chronic GVHD was mainly determined by graft source and in vivo TCD. In CR1, age, female donor to male recipient and donor type were additional factors (supplemental Tables 9-10 or details).

\section{Matched-pair analysis}

To confirm the results from the Cox model, a matchedpair comparison was performed between de novo and sAML. Using the criteria mentioned above, 877 wellmatched pairs were identified (cf. supplemental Table S11). The matched-pair analysis confirmed the findings of the Cox model: Overall, sAML was associated with a lower 3-year cumulative incidence of OS of $46.9 \%$ (95\% CI, 43.3-50.5) compared to $53.9 \%$ (95\% CI, 50.1-57.7) in de novo AML $(p=0.01)$. Furthermore, patients with sAML had a lower LFS $(p=0.03)$, GRFS $(p=0.04)$, and a higher NRM $(p=0.01)$. However, when the analysis was stratified by disease status at alloSCT, again differences were only seen among patients transplanted in CR1 (719 pairs, $82 \%$ ). The 3-year OS in the CR1 sAML and in the de 


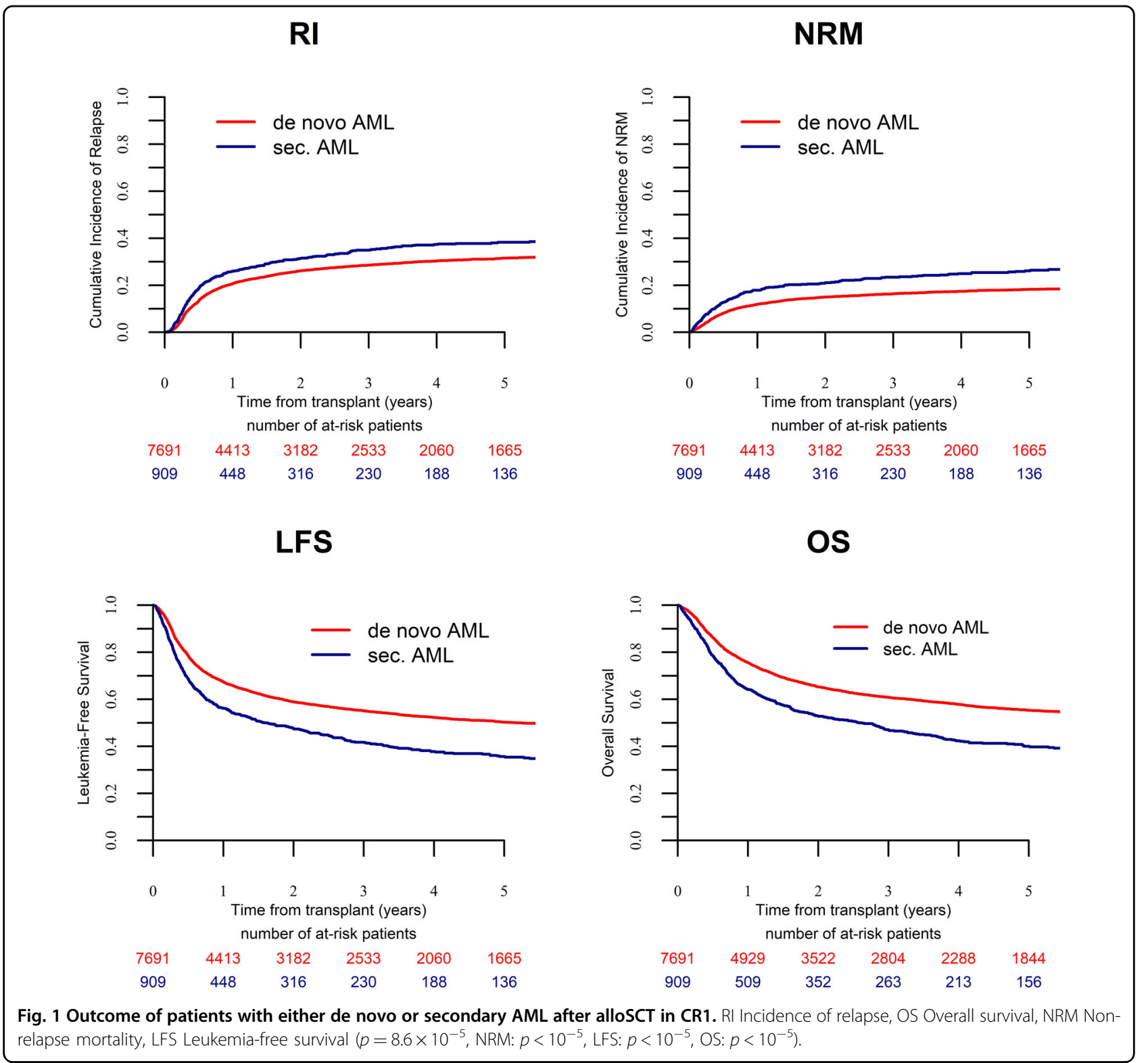

novo AML cohort was 48.1\% (95\% CI, 44-52.1) and 57.4\% (95\% CI, 53.2-61.6) ( $p=0.0004)$, respectively. In contrast, no significant difference in OS was observed between secondary and de novo AML after alloSCT in PIF (67 pairs, $7.6 \%, p=0.58$ ) and relapse (44 pairs, $5 \%, p=0.20$ ). Results of the matched pair analysis are shown in Table 4.

\section{Discussion}

In this large-scale, registry-based analysis, a multivariate model identified SAML as an independent risk factor for OS, LFS, GRFS, RI, and NRM after alloSCT in CR1, as compared to de novo disease. A matched-pair analysis confirmed these findings, which were observed both in treatment-related AML and AML evolving from other myeloid disorders or BM failure syndromes. In contrast, in patients with active disease at alloSCT, with relapse being by far the most frequent cause of treatment failure, the classical risk factors such as cytogenetics, age and KPS determined outcome. Secondary AML had no influence on outcome in the context of the aggressive nature of advanced disease.

Patients diagnosed with SAML tend to be of older age, carry more unfavourable cytogenetics and show poor response to chemotherapy ${ }^{20,21}$. Regardless of cytogenetics and preceding therapy of antecedent haematological diseases, SAML is known to have a poor long-term remission even when using aggressive induction therapy ${ }^{22,23}$. Separate studies on patients with treatment-related AML following intensive chemotherapy, identified tAML as an independent adverse prognostic factor ${ }^{2,7,14,24}$. However, 
Table 2 OS, Relapse, NRM, LFS, and GRFS at 3 years (univariate analysis).

\begin{tabular}{|c|c|c|c|c|}
\hline & & CR1 & PIF & Rel \\
\hline \multirow[t]{3}{*}{ OS } & de novo AML & $60.8 \%$ [59.6-62] & $29.8 \%$ [25.6-34] & $23.6 \%[20.7-26.4]$ \\
\hline & sAML & $46.7 \%[43.1-50.3]$ & $35.7 \%[28.5-42.9]$ & $24.8 \%$ [16.7-33] \\
\hline & $p$ (log rank) & $<10^{-5}$ & 0.541 & 0.721 \\
\hline \multirow[t]{3}{*}{ Relapse } & de novo AML & $28.5 \%$ [27.4-29.6] & $53 \%$ [48.6-57.3] & $58.4 \%[55.1-61.5]$ \\
\hline & sAML & $35 \%[31.7-38.4]$ & $43 \%[35.6-50.1]$ & $49.5 \%$ [40-58.2] \\
\hline & $p$ (log rank) & $8.6 \times 10^{-5}$ & 0.05 & 0.089 \\
\hline \multirow[t]{3}{*}{ NRM } & de novo AML & $16.4 \%[15.5-17.3]$ & $21.2 \%$ [17.9-24.7] & $23 \%[20.3-25.7]$ \\
\hline & sAML & $23.4 \%[20.5-26.4]$ & $27.9 \%[21.5-34.6]$ & $30.3 \%[22.2-38.7]$ \\
\hline & $p$ (log rank) & $<10^{-5}$ & 0.027 & 0.115 \\
\hline \multirow[t]{3}{*}{ LFS } & de novo AML & $55.1 \%[53.8-56.3]$ & $25.8 \%$ [21.9-29.7] & $18.7 \%[16.1-21.3]$ \\
\hline & sAML & $41.6 \%$ [38-45.1] & $29.1 \%$ [22.3-36] & $20.3 \%$ [12.8-27.8] \\
\hline & $p$ (log rank) & $<10^{-5}$ & 0.753 & 0.674 \\
\hline \multirow[t]{3}{*}{ GRFS } & de novo AML & $38.6 \%$ [37.4-39.9] & $16.8 \%[13.5-20.2]$ & $13.8 \%[11.6-16.1]$ \\
\hline & sAML & $28.4 \%[25.1-31.6]$ & $16.2 \%$ [10.5-21.9] & $16.3 \%$ [9.5-23.2] \\
\hline & $p$ (log rank) & $<10^{-5}$ & 0.981 & 0.448 \\
\hline
\end{tabular}

OS overall survival, NRM non-relapse mortality, LFS leukemia-free survival, GRFS Graft-versus-Host Disease/relapse-free survival, CR complete remission, PIF primary induction failure, $R e l$ relapse.

data on the role of sAML as a risk factor for outcome after alloSCT are scarce. Michelis et al. reported on a total of 264 patients with SAML and de novo AML, transplanted in CR1 $1^{12}$. Age, hematopoietic cell transplant comorbidity index, and karyotype were considered for matching. In contrast to our results, no clear influence of having sAML could be demonstrated regarding OS, NRM, LFS, and RI after alloSCT, using multivariate analysis and a propensity score model. It can be speculated, that the lower patient numbers precluded Michelis at al. from detecting a statistically significant difference $(p=0.18)$, given that the 3 -year OS in their cohort was $55 \%$ for de novo and $46 \%$ for sAML.

Besides the classical risk factors, i.e., cytogenetics and age, as well as donor type, our data identify sAML as an independent intrinsic factor for inferior outcome after alloSCT in CR1. Both patient and disease specific factors might contribute to this finding. First, characteristics associated with NRM (i.e., age, HLA mismatch, female donor to male patient, CMV seropositivity) had a high impact on OS in patients with sAML. This observation could suggest a lower ability to generally tolerate side effects and complications in the context of alloSCT, such as toxicity of the conditioning and immunosuppression, infections and GVHD. It might be a consequence of prior chemotherapy given for the antecedent hematologic or solid organ tumors, and might not be reflected by the performance score at time of alloSCT among patients with $\mathrm{SAML}^{21,25}$. Second, given the higher number of patients with sAML who have adverse cytogenetics, indicating lower sensitivity to standard chemotherapy and hypomethylating agents, the quality of CR might generally be inferior in SAML as compared to de novo disease. A better remission quality, however, is decisive for the outcome after alloSCT in sAML ${ }^{26}$, as well as de novo $\mathrm{AML}^{27,28}$. Third, the more aggressive nature of a secondary malignancy as such might play a role in the inferior outcome of patients with sAML, when transplanted in CR1.

Previous reports have suggested, that AML following antineoplastic radiation or chemotherapy (tAML) be considered an independent entity ${ }^{25}$. Although there are no pathognomonic genetic aberrations known for tAML, this subgroup had a worse outcome than MDS-related AML in several studies ${ }^{2,7,14,24}$. In a previous study from our group, slight differences in NRM and survival had been observed between patients with AML evolving from MDS/MPN and other hematologic malignancies, but not solid tumors ${ }^{8}$. In the present analysis, we therefore repeated the Cox model including tAML and AML evolving from another hematologic disease, as covariates. Both subgroups achieved comparable outcomes and were associated with increased OS, LFS and GRFS following alloSCT in CR1. Hence, as suggested earlier, the described two subtypes might be grouped as SAML in the setting of alloSCT ${ }^{12}$, although our numbers were to small to compare outcome of sAML following MDS versus sAML versus MPN. 
Table 3 Multivariate analysis of risk factors for overall survival, stratified by stage at transplantation.

\begin{tabular}{|c|c|c|c|c|c|c|c|c|c|}
\hline & \multicolumn{3}{|c|}{ SCT in CR1 } & \multicolumn{3}{|c|}{$\mathrm{SCT}$ in PIF } & \multicolumn{3}{|c|}{$\mathrm{SCT}$ in relapse } \\
\hline & HR & $\mathrm{Cl}$ & $p$ & HR & $\mathrm{Cl}$ & $p$ & HR & $\mathrm{Cl}$ & $p$ \\
\hline SAML & 1.33 & $1.21-1.48$ & $<10^{-5}$ & 0.917 & $0.726-1.157$ & 0.46499 & 0.946 & $0.752-1.192$ & 0.640 \\
\hline Age (per 10 years) & 1.18 & $1.15-1.225$ & $<10^{-5}$ & 1.103 & $1.025-1.188$ & 0.009 & 1.051 & $0.994-1.112$ & 0.081 \\
\hline Year of SCT & 0.996 & $0.987-1.006$ & 0.441 & 0.971 & $0.949-0.994$ & 0.015 & 0.999 & $0.981-1.018$ & 0.919 \\
\hline Relapse 2 vs relapse 1 & n.a. & & & n.a. & & & 1.232 & $1.029-1.475$ & 0.023 \\
\hline Favorable cytogenetics (ref) & 1 & & & 1 & & & 1 & & \\
\hline Intermediate & 1.27 & $1.088-1.482$ & 0.002 & n.a. & n.a. & & n.a. & & \\
\hline Adverse & 1.996 & $1.696-2.349$ & $<10^{-5}$ & 1.653 & $1.374-1.988$ & $<10^{-5}$ & 1.368 & $1.163-1.609$ & $<10^{-4}$ \\
\hline Female donor to male recipient & 1.124 & $1.033-1.224$ & 0.007 & 1.255 & $1.013-1.555$ & 0.037 & 1.155 & $0.972-1.373$ & 0.102 \\
\hline Previous autograft & 1.371 & $1.012-1.857$ & 0.041 & n.a. & & & n.a. & & \\
\hline KPS $>80 \%$ & 0.662 & $0.555-0.789$ & $<10^{-5}$ & 0.517 & $0.405-0.66$ & $<10^{-5}$ & 0.56 & $0.464-0.676$ & $<10^{-5}$ \\
\hline MSD (ref) & 1 & & & 1 & & & 1 & & \\
\hline UD 10/10 & 1.135 & $1.036-1.243$ & 0.007 & 1.043 & $0.809-1.345$ & 0.746 & 0.806 & $0.668-0.973$ & 0.025 \\
\hline UD 9/10 & 1.361 & $1.189-1.558$ & $10^{-5}$ & 0.98 & $0.7-1.371$ & 0.904 & 1.17 & $0.933-1.468$ & 0.174 \\
\hline Haploidentical donor & 1.333 & $1.11-1.602$ & 0.002 & 1.304 & $0.905-1.88$ & 0.154 & 0.912 & $0.705-1.179$ & 0.482 \\
\hline $\mathrm{PB}$ vs $\mathrm{BM}$ & 1.002 & $0.907-1.106$ & 0.976 & 0.93 & $0.672-1.286$ & 0.660 & 0.804 & $0.648-0.998$ & 0.048 \\
\hline Patient CMV-positive & 1.094 & $1.011-1.183$ & 0.025 & 1.24 & $1.006-1.53$ & 0.044 & 1.028 & $0.874-1.209$ & 0.738 \\
\hline Donor CMV-positive & 0.968 & $0.899-1.042$ & 0.386 & 1.048 & $0.861-1.275$ & 0.643 & 1.101 & $0.947-1.28$ & 0.209 \\
\hline MAC vs RIC & 1.026 & $0.94-1.12$ & 0.570 & 1.01 & $0.823-1.24$ & 0.923 & 1.062 & $0.91-1.239$ & 0.448 \\
\hline In vivo T-cell depletion & 0.935 & $0.859-1.018$ & 0.122 & 0.887 & $0.703-1.119$ & 0.311 & 1.108 & $0.939-1.308$ & 0.223 \\
\hline
\end{tabular}

SCT stem cell transplantation, $C R$ complete remission, PIF primary induction failure, $H R$ hazard ratio, $C l$ confidence interval, ref reference, $K P S$ Karnofsky performance status, MSD matched sibling donor, UD unrelated donor, $P B$ peripheral blood, BM bone marrow, $C M V$ cytomegalovirus, MAC myeloablative conditioning, RIC reducedintensity conditioning.

Statistically significant values are marked in bold.

Due to the retrospective nature of our study, some limitations, including missing information on MRD status and pre- and post-transplant therapies in most patients, must be acknowledged. In addition, there is a risk of selection bias, as patients without information on cytogenetics were deliberately excluded from this analysis, considering the high relevance of cytogenetic subgroups for outcome. Nevertheless, overall outcome data in our cohort are comparable to data from other studies ${ }^{8}$, suggesting that the cohort was representative. It must also be acknowledged that information on molecular aberrations was available only in a minority of patients from both cohorts and could therefore not be considered. However, in contrast to cytogenetic aberrations, which without doubt have similarly strong prognostic value in both de novo and sAML, less is known on the comparable value of molecular markers in both subgroups, which is why inclusion of these markers in this comparison would be difficult. Further, in the matched-pair analysis, patients with sAML and especially those in CR2 are most probably underrepresented, and fewer patients than expected could finally be included in the model. Therefore, the matchedpair analysis can only be regarded as confirmatory for the overall results obtained from the Cox model, which included all patients, and failed to reproduce the increased risk of relapse in SAML, most likely due to limited numbers.

This large-scale retrospective study identified SAML as an independent risk factor for alloSCT in CR1. Both the inferior quality of $\mathrm{CR}$ and higher sensitivity to toxicity might have contributed to the inferior outcome of sAML. These data help to improve risk stratification and prognostic estimates after alloSCT for sAML. Furthermore, the results may contribute to the design of optimized transplant protocols. Previous studies have suggested comparable outcomes after RIC and MAC in sAML, however, with a trend for better outcome after MAC ${ }^{11,29}$. Hence, a myeloablative, but reduced toxicity conditioning regimen such as the combination of Fludarabine and Treosulfan ${ }^{30}$ might be of particular value in patients with 
Table 4 OS, Relapse, NRM, LFS, and GRFS at 3 years according to matched-pair analysis.

\begin{tabular}{llllll}
\hline & & Entire cohort & CR1 & PIF & Rel \\
\hline OS & De novo AML & $53.9 \%[50.1-57.7]$ & $57.4 \%[53.2-61.6]$ & $32.4 \%[20.1-44.7]$ & $23.9 \%[9.5-38.3]$ \\
& SAML & $46.9 \%[43.3-50.5]$ & $48.1 \%[44-52.1]$ & $36.3 \%[23.4-49.2]$ & $33.5 \%[19.2-47.9]$ \\
& HR (95\% CI) $p$ value & $1.25(1.05-1.49) p=0.01$ & $1.43(1.17-1.74) p<10^{-3}$ & $0.86(0.50-1.48) p=0.58$ & $0.65(0.34-1.25) p=0.20$ \\
Relapse & De novo AML & $34.3 \%[30.9-37.8]$ & $32.4 \%[28.6-36.3]$ & $49 \%[35.5-61.1]$ & $53.7 \%[37.3-67.6]$ \\
& SAML & $36.9 \%[33.5-40.3]$ & $35.9 \%[32.1-39.7]$ & $45.8 \%[32.5-58.1]$ & $46.8 \%[31-61.1]$ \\
& HR (95\% Cl) $p$ value & $1.08(0.88-1.33) p=0.44$ & $1.24(0.98-1.56) p=0.07$ & $0.48(0.24-0.96) p=0.04$ & $0.87(0.41-1.82) p=0.71$ \\
NRM & De novo AML & $16.6 \%[14-19.4]$ & $15.7 \%[12.9-18.8]$ & $20.6 \%[11.2-32]$ & $23.6 \%[10.8-39]$ \\
& SAML & $21.3 \%[18.5-24.2]$ & $21.2 \%[18.1-24.4]$ & $24.4 \%[14.2-36.1]$ & $21.4 \%[10.4-35]$ \\
& HR (95\% Cl) $p$ value & $1.42(1.08-1.87) p=0.01$ & $1.47(1.08-2.0) p=0.01$ & $1.57(0.61-4.05) p=0.35$ & $0.83(0.25-2.73) p=0.76$ \\
LFS & De novo AML & $49.1 \%[45.4-52.8]$ & $51.8 \%[47.7-56]$ & $30.4 \%[18.4-42.5]$ & $22.7 \%[8.8-36.7]$ \\
& SAML & $41.8 \%[38.3-45.4]$ & $42.9 \%[39-46.9]$ & $29.8 \%[17.7-41.9]$ & $31.8 \%[17.6-46]$ \\
& HR (95\% Cl) $p$ value & $1.19(1.01-1.40) p=0.03$ & $1.32(1.10-1.59) p=0.003$ & $0.72(0.42-1.23) p=0.23$ & $0.86(0.46-1.61) p=0.63$ \\
GRFS & De novo AML & $34.3 \%[30.7-38]$ & $37 \%[32.9-41]$ & $18.9 \%[8.4-29.4]$ & $7.5 \%[0-19.1]$ \\
& SAML & $28.6 \%[25.3-32]$ & $29.2 \%[25.4-32.9]$ & $18.6 \%[7.8-29.4]$ & $24.8 \%[11.6-37.9]$ \\
& HR (95\% Cl) $p$ value & $1.13(0.97-1.32) p=0.12$ & $1.21(1.02-1.44) p=0.03$ & $0.82(0.49-1.36) p=0.44$ & $0.86(0.47-1.60) p=0.64$ \\
\hline
\end{tabular}

OS overall survival, NRM non-relapse mortality, LFS leukemia-free survival, GRFS Graft-versus-Host Disease/relapse-free survival, HR hazard ration, CI confidence interval, $C R$ complete remission, PIF primary induction failure, $R e l$ relapse.

sAML. In addition, strategies to improve the quality of remission prior to alloSCT in those patients with frequently low sensitivity to chemotherapy might be of particular importance ${ }^{31}$.

\section{Acknowledgements}

Following EBMT publication rules, co-authorship was offered to centers contributing the highest number of patients. We also highly appreciate the contribution by many physicians and data managers throughout the EBMT, who made this analysis possible, as well as the contribution by the patients themselves. A list of contributing centers and responsible physicians is provided in supplemental Table 12.

\section{Author details}

'Department of Hematology and Oncology, Augsburg University Hospital, Augsburg, Germany. ${ }^{2}$ Department of Haematology, Saint Antoine Hospital, Université Pierre et Marie Curie, INSERM UMR 938 Paris, France. ${ }^{3}$ EBMT Paris study office/ CEREST-TC, Paris, France. ${ }^{4}$ Hopital St. Louis, Dept.of Hematology BMT, Paris, France. ${ }^{5} \mathrm{HUCH}$ Comprehensive Cancer Center, Stem Cell Transplantation Unit, Helsinki, Finland. ${ }^{6}$ Programme de Transplantation \& Therapie Cellulaire, Centre de Recherche en Cancérologie de Marseille, Institut Paoli Calmettes, Marseille, France. ${ }^{7} \mathrm{CHU}$ de Lille, LIRIC, INSERM U995, Université de Lille, 59000 Lille, France. ${ }^{8} \mathrm{CHU}$ Bordeaux Hôpital Haut-Leveque, Pessac, France. ${ }^{9}$ Erasmus MC Cancer Institute, University Medical Center Rotterdam, Department of Hematology, Rotterdam, The Netherlands. ${ }^{10}$ Hannover Medical School, Department of Haematology, Hemostasis, Oncology and Stem Cell Transplantation, Hannover, Germany. ${ }^{11}$ University Hospital, Department of Bone Marrow Transplantation, Essen, Germany. ${ }^{12}$ Centre Hospitalier Lyon Sud, Service Hematologie, Lyon, France. ${ }^{13}$ University Hospital, Hematology, Basel, Switzerland. ${ }^{14}$ Division of Hematology/Oncology, Vanderbilt University Medical Center, Nashville, TN, USA. ${ }^{15}$ Hematology Division, Chaim Sheba Medical Center, Tel Hashomer, Israel

\section{Conflict of interest}

The authors declare that they have no conflict of interest.

\section{Publisher's note}

Springer Nature remains neutral with regard to jurisdictional claims in published maps and institutional affiliations.

Supplementary Information accompanies this paper at (https://doi.org/ 10.1038/s41408-020-0296-3).

Received: 5 September 2019 Accepted: 12 December 2019 Published online: 03 March 2020

\section{References}

1. Campo, E. et al. The 2008 WHO classification of lymphoid neoplasms and beyond: evolving concepts and practical applications. Blood 117, 5019-5032 (2011).

2. Kayser, S. et al. The impact of therapy-related acute myeloid leukemia (AML) on outcome in 2853 adult patients with newly diagnosed AML. Blood 117, 2137-2145 (2011).

3. Shin, S. H. et al. Survival benefits with transplantation in secondary AML evolving from myelodysplastic syndrome with hypomethylating treatment failure. Bone Marrow Transplant. 48, 678-683 (2013).

4. de Witte, T. et al. Intensive chemotherapy for poor prognosis myelodysplasia (MDS) and secondary acute myeloid leukemia (SAML) following MDS of more than 6 months duration. A pilot study by the Leukemia Cooperative Group of the European Organisation for Research and Treatmen. Leukemia 9, 1805-1811 (1995).

5. Hulegårdh, E. et al. Characterization and prognostic features of secondary acute myeloid leukemia in a population-based setting: A report from the Swedish Acute Leukemia Registry. Am. J. Hematol. 90, 208-214 (2015).

6. Borthakur, G. et al. Survival is poorer in patients with secondary core-binding factor acute myelogenous leukemia compared with de novo core-binding factor leukemia. Cancer 115, 3217-3221 (2009).

7. Schoch, C., Kern, W., Schnittger, S., Hiddemann, W. \& Haferlach, T. Karyotype is an independent prognostic parameter in therapy-related acute myeloid leukemia (t-AML): An analysis of 93 patients with t-AML in comparison to 1091 patients with de novo AML. Leukemia 18, 120-125 (2004). 
8. Sengsayadeth, S. et al. Transplant outcomes for secondary acute myeloid leukemia: Acute Leukemia Working Party of the European Society for Blood and Bone Marrow Transplantation Study. Biol. Blood Marrow Transplant. 24, 1406-1414 (2018).

9. Li, Z. et al. Haploidentical transplantation outcomes for secondary acute myeloid leukemia: Acute Leukemia Working Party (ALWP) of the European Society for Blood and Marrow Transplantation (EBMT) study. Am. J. Hematol. 93, 769-777 (2018).

10. Gyurkocza, B. et al. Nonmyeloablative allogeneic hematopoietic cell transplantation in patients with acute myeloid leukemia. J. Clin. Oncol. 28 2859-2867 (2010)

11. Sengsayadeth, S. et al. Conditioning intensity in secondary AML with prior myelodysplastic syndrome/myeloproliferative disorders: an EBMT ALWP study. Blood Adv. 2, 2127-2135 (2018).

12. Michelis, F. V. et al. Comparable outcomes post allogeneic hematopoietic cell transplant for patients with de novo or secondary acute myeloid leukemia in first remission. Bone Marrow Transplant. 50, 907-913 (2015).

13. Goldstone, A. et al. The importance of diagnostic cytogenetics on outcome in AML: analysis of 1,612 patients entered into the MRC AML 10 trial. Blood $\mathbf{9 2}$ 2322-2333 (1998)

14. Godley, L. A. \& Larson, R. A. Therapy-related myeloid leukemia. Semin. Oncol. 35, 418-429 (2008).

15. Döhner, $H$. et al. Diagnosis and management of AML in adults: 2017 ELN recommendations from an international expert panel. Blood 129, 424-447 (2017).

16. Holtan, S. G. et al. Composite end point of graft-versus-host disease-free, relapse-free survival after allogeneic hematopoietic cell transplantation. Blood 125, 1333-1338 (2015).

17. McCurdy, S. R. et al. Comparable composite endpoints after HLA-matched and HLA-haploidentical transplantation with post-transplantation cyclophosphamide. Haematologica 102, 391-400 (2017).

18. EBMT Manual. https://www.ebmt.org/sites/default/files/2019-05/M.

19. Pullarkat, V., Slovak, M. L., Kopecky, K. J., Forman, S. J. \& Appelbaum, F. R. Impact of cytogenetics on the outcome of adult acute lymphoblastic leukemia: results of Southwest Oncology Group 9400 study. Blood 111, 2563-2572 (2008).

20. Appelbaum, F. R. et al. Age and acute myeloid leukemia. Blood 107, 3481-3485 (2006).
21. Boddu, P. C. et al. Characteristics and outcomes of older patients with secondary acute myeloid leukemia according to treatment approach. Cancer 123, 3050-3060 (2017).

22. Rizzieri, D. A. et al. Outcomes of patients who undergo aggressive induction therapy for secondary acute myeloid leukemia. Cancer. 115, 2922-2929 (2009).

23. Schuler, E. et al. Long-term outcome of high risk patients with myelodysplastic syndromes or secondary acute myeloid leukemia receiving intensive chemotherapy. Ann. Hematol. 97, 2325-2332 (2018).

24. Borthakur, G. E. A. Therapy-related acute myelogenous leukemia and myelodysplastic syndrome. Curr. Oncol. Rep. 9, 373-377 (2007).

25. Østgård, L. S. G. et al. Epidemiology and clinical significance of secondary and therapy-related acute myeloid leukemia: A national population-based cohort study. J. Clin. Oncol. 33, 3641-3649 (2015).

26. Walter, R. B. et al. Comparison of minimal residual disease as outcome predictor for AML patients in first complete remission undergoing myeloablative or nonmyeloablative allogeneic hematopoietic cell transplantation. Leukemia 29, 137-144 (2015).

27. Balsat, M. et al. Postinduction minimal residual disease predicts outcome and benefit from allogeneic stem cell transplantation in acute myeloid leukemia with NPM1 mutation: a study by the acute leukemia French association group. J. Clin. Oncol. 35, 185-193 (2017).

28. Getta, B. M. et al. Multicolor flow cytometry and multigene next-generation sequencing are complementary and highly predictive for relapse in acute myeloid leukemia after allogeneic transplantation. Biol. Blood Marrow Transplant. 23, 1064-1071 (2017).

29. Lee, C. J. et al. Comparative outcomes of myeloablative and reduced-intensity conditioning allogeneic hematopoietic cell transplantation for therapy-related acute myeloid leukemia with prior solid tumor: a report from the acute leukemia working party of the European societ. Am. J. Hematol. 94, 431-438 (2019).

30. Beelen, D. W. et al. Final results of a prospective randomized multicenter phase III trial comparing treosulfan/fludarabine to reduced intensity conditioning with busulfan/fludarabine prior to allogeneic hematopoietic stem cell transplantation in elderly or comorbid patients. Blood 130, 521 (2017).

31. Lancet, J. E. et al. Cpx-351 (cytarabine and daunorubicin) liposome for injection versus conventional cytarabine plus daunorubicin in older patients with newly diagnosed secondary acute myeloid leukemia. J. Clin. Oncol. 36, 2684-2692 (2018). 